\title{
On the Coordinator's Rule for Fast Paxos
}

\author{
Gustavo M. D. Vieira* Luiz E. Buzato \\ Institute of Computing-UNICAMP \\ Caixa Postal 6176 \\ 13083-970 Campinas, São Paulo, Brasil \\ \{gdvieira, buzato\}@ic.unicamp.br
}

\begin{abstract}
Fast Paxos is an algorithm for consensus that works by a succession of rounds, where each round tries to decide a value $v$ that is consistent with all past rounds. Rounds are started by a coordinator process and consistency is guaranteed by the rule used by this process for the selection of $v$ and by the properties of process sets called quorums. We show a simplified version of this rule for the specific case where the quorums are defined by the cardinality of these process sets. This rule is of special interest for implementors of the algorithm.
\end{abstract}

\section{Introduction}

The problem of deciding a single value out of a set of values proposed by processes is known as the consensus problem. This problem is easy to solve in the absence of failures, but it is impossible to solve in an asynchronous distributed system even if a single process fails by permanently stopping [1]. A better approximation of the failures that processes of a real distributed system can suffer is the one where processes stop but may later recover. Unfortunately, the impossibility also holds for these systems. One of the ways to get around the impossibility is to design algorithms that do not violate their safety requirements while the system behaves asynchronously and are certain to make progress if the system behaves partially synchronously for periods long enough to satisfy the progress requirements. Designing consensus algorithms for the asynchronous crash-recovery model is a difficult task of practical interest and probably Paxos [2] and Fast Paxos [3] are the most studied solutions so far.

Fast Paxos solves the consensus problem through a succession of rounds that lead to the choice of the consensus value. In each round a distinguished process, the coordinator, is responsible for picking a single value using a rule that is based on quorums of processes. Quorums of successive rounds are used to guarantee that if a single value has been chosen or might ever be chosen in previous rounds then the same value is going to be chosen in the current round. Thus, quorums are fundamental to the correctness of Fast Paxos because they are ultimately responsible for the validity of consensus.

Lamport [3] shows how quorums can be characterized using the cardinality of sets of processes, defining what minimum number of processes represents a quorum. However, he defines the coordinator's rule in terms of quorum sets and general set operations. This complete characterization

*Financially supported by CNPq, under grant 142638/2005-6. 
of the coordinator's rule is perfect for the purposes of his work, but it does not address thoroughly the needs of a programmer who wants to implement it. Therefore, the main contributions of this paper are (i) an interpretation of the Fast Paxos coordinator's rule only in terms of the cardinalities of quorum sets, and (ii) its simplification. The simplified interpretation is efficient, easier to implement and test; it can help developers to create reliable implementations of Fast Paxos. This is important, as the use of Fast Paxos to build fault-tolerant applications is bound to require the execution of a very large number of consensus instances.

\subsection{Fast Paxos}

Before detailing the coordinator's rule, it is useful to give a very brief overview of Fast Paxos; a complete description of it can be found in [3]. The algorithm is easier to explain in terms of reactive agents that represent a role, such that a single process can enact multiple agents, with each one of them playing a different role. An agent can enact one of the following main roles: a proposer that can propose values by sending them to acceptors, an acceptor that chooses a single value, or a learner that learn what value has been chosen.

To solve consensus, Fast Paxos agents execute multiple rounds, each round has a coordinator and may be either a fast round or a classic round. Positive integers are used to uniquely identify rounds, each identifier determines the coordinator and indicates the round type: fast or classic. Regardless of its type, each round progresses through two phases with two steps each:

- In Phase 1a the coordinator sends a message requesting every acceptor to participate in round $i$. An acceptor accepts the invitation if it has not already accepted to participate in round $j \geq i$, otherwise it declines the invitation by simply ignoring it.

- In Phase $1 \mathrm{~b}$ every acceptor that has accepted the invitation answers to the coordinator with a reply that contains the round number and the value of the last vote it has cast for a value, or null if it has not voted.

- In Phase 2a, if the coordinator of round $i$ has received answers from a quorum of acceptors then it executes its rule on the set of values suggested by acceptors in Phase $1 \mathrm{~b}$ and picks a single value $v$. It then asks the acceptors to cast a vote for $v$ in round $i$, if $v$ is not null, otherwise, if the round is fast the coordinator sends a any message to the proposers indicating that any value can be chosen in round $i$. In this case, the proposers ask the acceptors to cast a vote for a value $v$ of their choice in round $i$.

- In Phase $2 \mathrm{~b}$, after receiving a request to cast a vote from the coordinator or from one of the proposers, acceptors can either cast a vote for $v$ in round $i$, if they have not voted in any round $j \geq i$, otherwise, they ignore the vote request. Votes are cast by sending them together with the round identifier to the learners.

- Finally, a learner learns that a value $v$ has been chosen if, for some round $i$, it receives Phase $2 \mathrm{~b}$ messages from a quorum of acceptors announcing that they have all voted for $v$ in round $i$.

As Fast Paxos agents may crash and recover, they must save their state in stable memory so that agents, once recovered, can remember the votes they have cast earlier. The sequence of steps described above imply that a learner can only learn the value of consensus after a period of at least four message delays. If numerous executions of Fast Paxos are required, then it is possible to run 
Phase 1 and Phase 2a only once for all these instances. This factorization of phases is carried out immediately after the election of a coordinator. At this point, most of the consensus instances have not been started yet, allowing the coordinator to send Phase 2a any messages. The improvement brought about by this factorization allows consensus in two message delays, making Fast Paxos an optimal consensus algorithm [4]. Unfortunately, Fast Paxos cannot always be fast. Proposers can propose two different values concurrently, in this case, their proposals may collide. Also, process and communication failures may block a round from succeeding. Different recovery mechanisms can be implemented to deal with collisions and failures, but eventually the coordinator intervention may be necessary to start a new round [3]. Any process can act as the coordinator as long as it follows the rule for choosing a value, if any, that is proposed in Phase 2a.

As already mentioned, quorums are fundamental for Fast Paxos. Quorums are set of processes and each round has a set of quorums associated with it, classic quorums for a classic round and fast quorums for a fast round. For the proper operation of the algorithm, quorums have to satisfy properties on the sets of processes that form them. Specifically, any two quorum sets must have a non-empty intersection and any quorum and any two fast quorums from the same round must also have a non-empty intersection [3]. There are many ways to define quorums, but a very interesting one from the point of view of process fault tolerance is the definition based only on the number of processes contained in each quorum. The definition of quorum using this parameter is straightforward and is described in Section 2.

The coordinator's rule determines how a coordinator can consistently start a new round, after collecting information about previous rounds from the acceptors. That is, for each round $i$ the coordinator is about to start, it must know if a value $v$ had been decided or might have been decided in previous rounds $j<i$. The coordinator's rule of Fast Paxos must take into account that in a fast round, more than one value might have been proposed and voted concurrently. Quorums are defined to guarantee that only one, if any, of the conflicting proposed values is selected through the application of the coordinator's rule. Section 3 presents the original coordinator's rule as defined in [3] and then shows how this rule can be effectively implemented using a cardinality-based definition of quorums. Section 4 brings our derivation of a simplified cardinality-based coordinator rule, it is stricter than the one presented in Section 3, but it is easier to understand and to implement. Section 5 closes the work by commenting on the practical value of our main result: a simplified coordinator's rule for Fast Paxos.

\section{Choosing Quorums}

The quorum requirements for Fast Paxos assert that: (a) any two quorums must have non-empty intersection, (b) any two fast quorums and any classic or fast quorum from the same round have a non-empty intersection [3]. We can satisfy these conditions by considering only the number of process in each quorum, where $N$ is the number of acceptors, and $F$ and $E$ are the maximum number of failed acceptors in classic and fast rounds, respectively [3]. A classic quorum is formed by $N-F$ acceptors and $N-E$ acceptors form a fast quorum. As the requirements for fast quorums are always stricter than those for classic quorums, we can always assume that $E \leq F$. The quorum conditions [3] are then stated as:

$$
\begin{aligned}
& N>2 F \\
& N>2 E+F
\end{aligned}
$$


For a fixed $N, F$ and $E$ can be chosen in various different ways and a natural way of choosing them is by maximizing one or the other [3]. As we have $E \leq F$, maximizing $E$ leads to $E=F$. Thus, we can satisfy the system only with $N>3 F$ and:

$$
N>3 F \Leftrightarrow F<N / 3 \Leftrightarrow F \leq\lceil N / 3\rceil-1
$$

For this case, the cardinality of any classic quorum $\left(\left|Q_{c}\right|\right)$ or fast quorum $\left(\left|Q_{f}\right|\right)$, expressed only as a function of $N$, is:

$$
\left|Q_{c}\right|=\left|Q_{f}\right| \geq N-\lceil N / 3\rceil+1 \geq\lfloor 2 N / 3\rfloor+1
$$

If instead we maximize $F$, the limit for its value is given by the Equation 1, thus:

$$
N>2 F \Leftrightarrow F<N / 2 \Leftrightarrow F \leq\lceil N / 2\rceil-1
$$

In this case $E$ must be chosen to satisfy Equation 2, considering the value of $F$ we have just chosen:

$$
N>2 E+F \Leftrightarrow N>2 E+\lceil N / 2\rceil-1 \Leftrightarrow 2 E \leq N-\lceil N / 2\rceil \Leftrightarrow E \leq\lfloor N / 4\rfloor
$$

For this case, the cardinality of any classic quorum $\left(\left|Q_{c}\right|\right)$ and fast quorum $\left(\left|Q_{f}\right|\right)$, expressed only as a function of $N$, is:

$$
\begin{array}{r}
\left|Q_{c}\right| \geq N-\lceil N / 2\rceil+1 \geq\lfloor N / 2\rfloor+1 \\
\left|Q_{f}\right| \geq N-\lfloor N / 4\rfloor \geq\lceil 3 N / 4\rceil
\end{array}
$$

\section{Coordinator's Rule}

The original coordinator's rule for Fast Paxos [3] is:

LET $Q$ be any $i$-quorum of acceptors that have reported their last votes to the coordinator.

$v r(a)$ and $v v(a)$ be the round and the value voted by acceptor $a$.

$k$ be the largest value of $\operatorname{vr}(a)$ for all $a \in Q$.

$V$ be the set of values $v v(a)$ for all $a \in Q$ with $v r(a)=k$.

$O 4(v)$ be true iff there is a $k$-quorum $R$ such that $\operatorname{vr}(a)=k$ and $v v(a)=v$ for all $a \in(Q \cap R)$.

IF $k=0$ THEN let $v$ be any proposed value.

ELSE IF $V$ contains a single element

THEN let $v$ equal that element.

ELSE IF there is some $w \in V$ satisfying $O 4(w)$

THEN let $v$ equal that $w$ (unique).

ELSE let $v$ be any proposed value.

We now show how this rule can be interpreted in terms of the cardinality-based quorum definitions presented in the previous section. When $k=0$ or $V$ contains a single element the rule is trivial to evaluate no matter the quorum implementation used. However, the evaluation of $O 4(w)$ is more complex because it requires the evaluation of all possible intersections $Q \cap R$ for all $k$-quorums 
$R$. Considering only the cardinality of the quorums involved we have that $O 4(w)$ is true if at least $|Q \cap R|$ acceptors voted for $w$ for some $R$. As we don't know, and don't want to know, all possible quorums $R$, we must consider the smallest possible $|Q \cap R|$, assuming as implied by $O 4(w)$ that all acceptors outside of $Q$ also voted for $w$ in ballot $k$. Considering that $V$ can only contain more than one element if $k$ was a fast round, we have two situations: $i$ is a classic quorum or $i$ is a fast quorum. Let $T$ be the number of votes for the value $w$ in $V$. If we want $T$ to be at least as large as the smallest $|Q \cap R|$ then we have:

$$
T \geq \begin{cases}N-E-F & \text { if } i \text { is classic } \\ N-2 E & \text { if } i \text { is fast }\end{cases}
$$

$O 4(w)$ can now be evaluated by simply counting the number of votes for $w$ in $V$. So, any value $w$ that satisfies the condition above satisfies $O 4(w)$ and is by definition unique. Considering that $E<(N-F) / 2$ from Equation 1, when $i$ is a classic round, we have:

$$
T \geq N-E-F \Rightarrow T>N-(N-F) / 2-F \Leftrightarrow T>(N-F) / 2 \Leftrightarrow T \geq\left\lfloor\left|Q_{c}\right| / 2\right\rfloor+1
$$

Similarly, for the case where $i$ is a fast round we have:

$$
\begin{aligned}
T \geq N-2 E & \Rightarrow T>N-E-(N-F) / 2 \Leftrightarrow 2 T>N-E+(F-E) \\
& \Rightarrow T>(N-E) / 2 \Leftrightarrow T \geq\left\lfloor\left|Q_{f}\right| / 2\right\rfloor+1
\end{aligned}
$$

In all cases $T$ is at least as large as $\lfloor|Q| / 2\rfloor+1$, so if any value $w$ satisfies $O 4(w)$, then it has been voted in round $k$ by a majority of processes inside the quorum $Q$.

\section{Simplified Coordinator's Rule}

We have shown that a value $w$ satisfies $O 4(w)$ if this value have been voted in round $k$ by a majority of acceptors in $Q$. We can use this observation to derive a simplified coordinator's rule for Fast Paxos. First, the fact that $w$ has been voted by a majority in $Q$ implies that it is the value most often voted in $V$. Thus, we can check this condition before testing if $w$ satisfies $O 4(w)$, obtaining an equivalent coordinator's rule.

IF $k=0$ THEN let $v$ be any proposed value.

ELSE IF $V$ contains a single element

THEN let $v$ equal that element.

ELSE IF there is a single $w \in V$ voted most often

THEN IF $w$ satisfies $O 4(w)$

THEN let $v$ equal that $w$.

ELSE let $v$ be any proposed value.

ELSE let $v$ be any proposed value.

If $w$ do not satisfy $O 4(w)$, we are free to choose any value as $v$. We use this freedom to always select the most often voted $w$. We have now removed some freedom from the coordinator, but all values $w$ that satisfy $O 4(w)$ are correctly selected. We can remove the $O 4(w)$ test, obtaining the following rule: 
IF $k=0$ THEN let $v$ be any proposed value.

ELSE IF $V$ contains a single element

THEN let $v$ equal that element.

ELSE IF there is a single $w \in V$ voted most often

THEN let $v$ equal that $w$.

ELSE let $v$ be any proposed value.

If $V$ contains a single element, then this element surely has been voted most often than any other element in $V$. Thus, we can remove the single element test, giving our final simplified rule:

IF $k=0$ THEN let $v$ be any proposed value.

ELSE IF there is a single $w \in V$ voted most often

THEN let $v$ equal that $w$.

ELSE let $v$ be any proposed value.

\section{Conclusion}

We have showed how the coordinator's rule of Fast Paxos [3] can be simplified by resorting exclusively to counting the number of votes for each of the proposed values. Our rule is more restrictive than the original rule, as for some consensus instances it forbids the coordinator of freely choosing any value when he would be allowed otherwise by the original rule. However, the restriction imposed by the simplification does not lead to any disadvantage because if some value received votes in a previous round and the consensus value isn't decided yet, it is reasonable to consider that the coordinator will try to decide on that value first. Our simplified rule is easier to implement, has the advantage that it is independent of the type of a round (fast or classic), and it has to consider only the cardinality of the quorum $Q$.

\section{References}

[1] Michael J. Fischer, Nancy A. Lynch, and Michael S. Paterson. Impossibility of distributed consensus with one faulty process. J. ACM, 32(2):374-382, 1985.

[2] Leslie Lamport. The part-time parliament. ACM Trans. Comput. Syst., 16(2):133-169, 1998.

[3] Leslie Lamport. Fast Paxos. Distrib. Comput., 19(2):79-103, October 2006.

[4] Leslie Lamport. Lower bounds for asynchronous consensus. Distributed Computing, 19(2):104125, June 2006. 